\title{
25 Research Square \\ CT features and clinical manifestations of ordinary pneumonia with COVID-19 infection: A multi-center study
}

\section{Kun-ming Yi}

Army Medical University

Liu Yu

People's Hospital of Kaizhou

Hai-peng Tong

Army Medical University

Yun Tian

People's Hospital of Wushan

Xiao-hui Li

People's Hospital of Dianjiang

Ran Li

People's Hospital of Banan

Jing-qin Fang ( $\nabla_{\text {jingqin0405@163.com ) }}$

Army Medical University

\section{Research Article}

Keywords: 2019 novel coronavirus disease (COVID-19); Pneumonia; Ordinary; Computed tomography.

Posted Date: April 20th, 2020

DOI: https://doi.org/10.21203/rs.3.rs-23394/v1

License: (c) (1) This work is licensed under a Creative Commons Attribution 4.0 International License. Read Full License 


\section{Abstract}

Purpose: Investigate clinical and CT manifestations of ordinary coronavirus disease (COVID-19) pneumonia.

Materials and methods: Patients with ordinary COVID-19 pneumonia (confirmed by RT-PCR) and performed initial chest CT were retrospectively enrolled. Fifty-eight patients were assigned to group 1 ( $<50$ years, $n=33)$ and group $2(50 \geq$ years, $n=25)$ on the basis of age. The clinical data, laboratory results, and imaging findings were evaluated. Imaging features were analyzed and compared across the two groups.

Results: On chest CT imaging, 44 (75.9\%) patients showed bilateral lung involvement, 14(24.1\%) showed unilateral lung involvement, with predominant peripheral distribution $(26 / 58,44.8 \%)$ and mixed distribution (30/58, 51.7\%). 445(445/634, 70.2\%) lesions occurred in the lung periphery, $189(29.8 \%)$ in the center. There were more lobes involved in group 2(median 4, IQR 3-5) than group 1(median 3, IQR 1.54) $(P=0.024)$. Ground glass opacity (GGO) $(451 / 634,71.1 \%)$ and consolidation $(157 / 634,24.8 \%)$ were the main CT findings. Lesions with a maximum diameter greater than $5 \mathrm{~cm}$ were more common in group 2 $(19 / 25,76 \%)$ than group $1(12 / 33,36.4 \%)(P=0.003)$. The $C T$ score of bilateral lungs, right lung, left lung and each lobe in group 2 was significantly higher than those of group 1 (all $P<0.05$ ), except for the middle lobe of the right lung $(P=0.979)$.

Conclusions: Ordinary COVID-19 pneumonia on chest CT generally manifested as multiple GGO and consolidation in the bilateral lung, with predominant peripheral or mixed distribution. Older age might be a risk factor for progression in ordinary COVID-19 pneumonia.

\section{Introduction}

In December 2019, China reports a cluster of cases of pneumonia with unknown etiology in Wuhan city, Hubei province to the World Health Organization (WHO). Then, a previously unknown betacoronavirus was discovered through unbiased sequencing in samples from patients with pneumonia[1]. This betacoronavirus was formally named severe acute respiratory syndrome coronavirus 2 (SARS-Cov-2) by the International Committee on Taxonomy of Viruses [2]. The disease caused by SARS-Cov-2 was termed corona virus disease 2019 (COVID-19) by WHO[3]. Approximately just in one month, COVID-19 rapidly spread from within Wuhan city to the entire country. By March 26, 2020, there are 82034 confirmed cases in China, 3294 patients died, 74204 patients were cured. Outside China, 404223 cases were confirmed in more than 180 countries. Patients with confirmed SARS-Cov-2 infection are the source of virus, even in the incubation period. Currently, the SARS-Cov-2 was confirmed to be propagated through droplet and close contact, aerosol transmission only could be possible in a closed environment. The onset of the COVID-19 outbreak near annual Lunar New Year, the most significant holiday in China, when people return to their hometown through crowded trains, buses, and planes. That means each COVID-19 infected patients could have numerous close contacts, which may be the main reason for the spreading of COVID19 outside the Wuhan city in a short time. 
Unlike SARS in 2002, most of the COVID-19 infected patients demonstrate mild symptoms, including fever, fatigue and dry cough, which could be mistaken as the common cold and cause overlook by patients. That prompt inadequate isolation and subsequent transmission of COVID-19. The final etiology diagnosis of COVID-19 is positive RT-PCR assay for SARS-Cov-2 nucleic acid in respiratory or blood samples or highly homologous with COVID-19 by viral gene sequencing of respiratory or blood samples. However, RT-PCR results sometimes are false negative which could be attributed to several factors, such as low virus load, sampling error and insufficient sensitivity of kits and so on[4, 5]. In some cases, Computed tomography (CT) exhibited pneumonia but multiple RT-PCR tests of nasopharyngeal or throat swabs test negative[4, 6, 7]. Fang et al. concluded a higher detection rate for initial CT than the first RTPCR by comparing the detection rate of initial chest CT and RT-PCR[6]. Under the circumstances, chest CT findings are recommended as the major evidence for clinically confirmed cases by the National Health and Health Commission of China in the trail fifth version of Diagnosis and Treatment Program of 2019 New Coronavirus Pneumonia. Although the term of clinical diagnosis is removed in the recent version[8], CT imaging also offers key information for the further diagnosis of COVID-19. Since most COVID-19 cases are ordinary type, early diagnosis and quarantine of those seem "common cold" patients is crucial to block transmission and prevent ordinary patients from progressing to severe even critical type. However, most clinical literature focused on the severe and critical type of COVID-19, there is relatively limited summary radiographic information of ordinary type. Therefore, in this present study, we retrospectively evaluated the clinical and chest CT imaging findings of 58 confirmed ordinary cases. We hope our findings could help to better identify the COVID-19 in the early stage.

\section{Materials And Methods}

\subsection{Study Subjects}

This multi-institutional retrospective study was approved by the Institutional Research Ethics Board at each institution and patient consent was waived due to the study's retrospective nature. Between January 16,2020 , and February 22, 2020, 63 patients with 2019-nCoV infection confirmed by real-time reversetranscription-polymerase chain- reaction (RT-PCR) were evaluated from multiple centers. Eventually, according to the guideline of the trail seventh version of Diagnosis and Treatment Program of 2019 New Coronavirus Pneumonia( 2019-nCoV) [8], a total of 58 ordinary patients who underwent chest CT scanning were enrolled in this study, five severe/critical patients were excluded from this study. The clinical data, including age, sexes, exposure history, symptoms, time from illness onset to hospital admission, and laboratory results were reviewed retrospectively.

\subsection{MDCT Protocol}

All included patients underwent chest non-contrast enhanced director computed tomography (MDCT) scanning by 64-row MDCT (Lightspeed/ Revolution, GE Healthcare, Milwaukee, WI, USA) or a 16-slice MDCT (Emition, Siemens, Erlangen, Germany) or a 16-row MDCT(Lightspeed, GE Healthcare, Milwaukee, WI, USA) system. Scan parameters were as follows: 100-120 kV, 150-300 mA, 1or 5-mm-thick slice 
reconstructions, $23-\mathrm{cm} \mathrm{FOV}$, and reconstruction matrix of $512 \times 512$. Scans were obtained from the thoracic inlet to the caudal tip of the liver. Axial image at 1 or $5 \mathrm{~mm}$ and coronal image at $5 \mathrm{~mm}$ were reconstructed for all patients. Lung and mediastinal windows were reviewed.

\subsection{Image Analysis}

All imaging data were conducted by two chest radiologists (K.Y., J.F., with 8 and 11 years of experience, respectively) who were blinded to patients' clinical data in interpreting chest $\mathrm{CT}$ images by consensus. The CT imaging features were analyzed considering the following terms: (1) distribution characteristics(e.g., periphery or central or mixed [periphery, defined as the location of the lesion in the outer one-third of the lung, or the lesion that showed spread from the periphery to the pulmonary hilum; otherwise it is considered as center], bilateral or unilateral, multifocal or unifocal,); (2) number of involved lobes and segments); (3) patterns of the lesion (e.g., ground glass opacity[GGO, defined as hazy opacity with preservation of bronchial and vascular margins, included mixed GGO], consolidation[defined as opacification that obscured underlying the margins of vessels and airway walls], crazy paving pattern[defined as GGO with associated interlobular septal thickening], vascular enlargement[defined as the diameter of the vessel of the lesion was thicker than that of the contralateral vessel], halo sign[defined as a complete or almost complete ring of ground glass around consolidation; conversely, called reverse halo sign], interlobular septal thickening, cavitation, air bronchogram sign, bronchial wall thickening); (4) other abnormalities (e.g., thoracic lymphadenopathy[defined as lymph node size of $\geq 10 \mathrm{~mm}$ in shortaxis], subpleural line, pleural thickening, and fibrous stripes).

A semi-quantitative CT score was used to quantify the extent of diseases on the basis of the area involved [9]. Each lobe of the lung was given a score: score $0,0 \%$ involvement; score 1 , less than $5 \%$ of the lobe; score $2,5 \%$ to $25 \%$ of the lobe; score $3,26 \%$ to $49 \%$ of the lobe; score $4,50 \%$ to $75 \%$ of the lobe; and score 5 , more than $75 \%$ of the lobe. There was a score of $0-5$ for each lobe, and the total CT score was calculated by summing the five lobe scores (0-25). In addition, the number of involved lung lobes and segments was counted. As a lesion was not connected with the other lesions, it was considered as one lesion. when a large lesion involved more than one lung segment, the lesion was assigned to the segment accounted for the largest proportion of the lesion. Both the subpleural line and each side of the pleural thickening were counted as one lesion. Bronchial wall thickening, crazy paving pattern, interlobular septal thickening, air bronchogram sign, halo sign, and reverse halo sign were identified as concomitant imaging manifestations.

\subsection{Statistical Analysis}

In our study, patients were assigned to group 1 ( $<50$ years) and group 2 ( $50 \geq$ years) on the basis of age. Statistical analyses were performed by using the SPSS analysis program (version 22.0, IBM). Quantitative variables were expressed as mean (SD) or median (IQR) values. Shapiro-Wilk tests were used to test the normality of quantitative variables. Non-parametric data were tested using the independent Mann-Whitney U test or Kruskal-Wallis one-way analysis of variance. Categorical variables were reported 
as frequency rates and percentages and compared by Chi-squared test between groups. A $P$ value of less than 0.05 was considered significant.

\section{Results}

\subsection{Clinical Characteristics}

In this study, 58 patients who had confirmed ordinary COVID-19 pneumonia were retrospectively enrolled. The clinical characteristics and laboratory results were exhibited in Table 1. The mean age of the patients was $44.7 \pm 13.8$ years (range13-70 years), without gender difference ( 28 men and 30 women). The median duration of symptoms from illness onset to hospitalization was 3 days (IQR 1.0-5.3; range, 1-20 days). At the onset of illness, the most common symptoms were fever (74.1\%) and dry cough (70.7\%). Two (3.4\%) patients had decreased oxygen saturation. Two (3.4\%) patients were asymptomatic, they were treated for exposure to infected patients. On admission, most patients demonstrated lymphopenia $(60.3 \%)$ and elevated level of C-reactive protein (60.3\%), some patients showed increased neutrophils ratio $(20.7 \%)$

\subsection{MDCT Findings}

Of the 58 patients included, all patients presented abnormal imaging findings on chest CT (Table 2). Forty-four (75.9\%) patients showed bilateral lung involvement, 14 (24.1\%) showed exclusively unilateral lung involvement and ten of them had only one lesion. A total of 634 lesions were found, 445/634 (70.2\%) lesions located in the lung periphery and 189/634 (29.8\%) in the center. More than half of the lesions $(339 / 634,53.5 \%)$ located in the lower lobes in 55 (94.8\%) patients, 249 (39.2\%) lesions in the upper lobes in 49 (84.8\%) patients. Although all lung lobes can be affected, the right lower lobe was most frequently involved. Our findings showed that peripheral distribution was more prevailing in group 2 than group $1(P=0.02)$, while, central lesions showed no significant differences between groups $(P=0.62)$ (Table 3). There were more lobes involved in group 2 than group $1(P=0.024)$ but without significant differences of involved segments between groups $(P=0.12)$ (Table 3$)$. The most common involved lung segment was posterior basal segment of the right lower lobe (74/634), followed by posterior segment of the right upper lobe (71/634), apical-posterior segment of the left upper lobe (58/634), and posterior basal segment of the left lower lobe (56/634).

On chest CT, the main findings were ground-glass opacity (GGO) $(451 / 634,71.1 \%)$ (Figure 1 ) and consolidation (157/634, 24.8\%) (Figure 2). Twenty-seven (46.6\%) patients had only GGO (171/634, $27.0 \%), 5$ (8.6\%) patients showed only consolidation (48/634, 7.6\%), and the others (44.8\%) presented mixed findings $(415 / 634,65.4 \%)$ (Figure 3$)$. GGO can be accompanied by a crazy paving pattern (Figure 1), interlobular septal thickening, bronchial wall thickening, reverse halo sign. Consolidation with halo sign (Figure 2) and consolidation or GGO with vascular enlargement might be noted (Figure 1). More than half of the GGO distributed in the lung periphery, and nearly $1 / 3$ of the consolidation in the lung center. In our series, 10 (10/33, 30.3\%) patients with 57 consolidations in group 1 , and 11 (11/25, 44.0\%) patients with 100 consolidations in group 2 . There were no significant differences in the occurrence rate and the 
median number of consolidation between the two groups ( $P=0.282, P=0.36$, respectively) (Table 3 ). $83(83 / 634,13.1 \%)$ lesions whose maximum diameter was greater than $5 \mathrm{~cm}$ were observed and this type of lesion was more likely to occur in group $2(19 / 25,76 \%)$ than in group $1(12 / 33,36.4 \%)(P=0.003)$ (Table $3)$. The largest lesion was usually located in the right lower lobe $(27 / 58,46.6 \%)$, followed by left lower lobe $(15 / 58,25.9 \%)$, and right upper lobe $(9 / 58,15.5 \%)$.

The radiological scores on admission were summarized (Figure 4), and the difference of CT scores between group 1 and group 2 was evaluated in Table 3. Whether before or after grouping, there was no significant difference in the total CT score between the right lung and left lung (all $P>0.1$ ). Moreover, the CT score of bilateral lungs, right lung and left lung in group 2 was significantly higher than those of group 1 (all $P<0.05$ ). Meanwhile, the CT score of each lobe in group 2 was also higher than that of group 1 (all $P<0.05)$, except for the middle lobe of the right lung $(P=0.979)$.

\section{Discussion}

In this study, we investigated 58 patients with confirmed ordinary COVID-19 pneumonia. The most common clinical symptoms were nonspecific, including fever and dry cough. Laboratory results were consistent with the characteristics of viral infection, such as lymphopenia, lymphocyte ratio decreased, and elevated levels of C-reactive protein. Our findings are similar to the results of previous studies [10, 11]. In our presentation, most patients presented only GGO or GGO with consolidation pattern, with peripheral and subpleural distribution in both lungs, and multiple lobes and segments were involved. Interlobular septal thickening, crazy paving pattern, bronchial wall thickening, reverse halo sign, vascular enlargement, and halo sign were observed.

In previous literature, the typical chest CT features of COVID-19 pneumonia, including multiple bilateral GGOs or GGOs with consolidations, prominent peripherally distribution, and posterior part and lower lobe predilection [5, 12-24]. The right lower lobes were most commonly involved due to the right bronchus is short and straight, and the virus might tend to attach this location and cause infection[19]. On chest CT of ordinary COVID-19 pneumonia in our cohort, most lesions (70.2\%) occurred in the lung periphery, the right lower lobe and the posterior basal segment of the right lower lobe were frequently infected $(29.5 \%$, $11.7 \%$, respectively), which are consistent with the results of those previous studies. Thus, it may be indicated that the distribution trend of lesions in the lung is similar in the types of COVID-19 pneumonia (ordinary, severe, and critically severe types). Additionally, there were more peripheral lesions in the elder group (age $\geq 50$ years) than the younger group (age $<50$ years) in our study.

In addition, ordinary COVID-19 pneumonia was mostly at the early stage and the severe and critically severe COVID-19 pneumonia were significantly older and had more lobes involved than ordinary COVID19 pneumonia[15, 23]. Similar findings of the elder patients tended to have more areas of lung involvement were also reported by Song et al[13]. Shi et al[19]. reported that symptomatic patients had more segments involved than symptomatic patients and the patients (CT scans done $>1$ to 2 weeks from symptom onset) had the highest mean number of involved segments. As well, our result also showed that 
more lobes involved in the elder group (age $\geq 50$ years) than the younger group (age $<50$ years ). Therefore, we considered that elder patients might have more lung lobes involvement and more peripheral lesions in both ordinary COVID-19 pneumonia and severe/critical COVID-19 pneumonia. The original lesion located in the peripheral area could extend to the pulmonary hilum in large lesions or when the disease aggravated[23]. In our study, a total of 31 patients with $83(13.1 \%)$ lesions with a maximum diameter greater than $5 \mathrm{~cm}$ were observed, and this type of lesion was more likely to occur in the elder group than in the younger group $(P=0.003)$. This finding also supports that the degree of lung injury in elder patients was more severe than younger patients.

As is well known, the chest CT manifestation of COVID-19 pneumonia changed rapidly and diversely at different stages[18, 19, 23], which are mainly related to pathogenesis. In our cohort, 27 (46.6\%) patients had only GGO $(171 / 634,27.0 \%)$, which consistent with the early manifestations that the GGO pattern was the predominant CT finding in the early stage of COVID-19 pneumonia [13, 16, 19, 24, 25], probably because at first the virus mainly affects the terminal bronchioles and their surrounding pulmonary parenchyma with alveolar edema and interstitial infiltration of pulmonary lobules. In the Shi et al[19]. study, the GGO pattern was the predominant CT finding throughout the course of the disease, and the GGO continued to decrease and a transition to consolidation and mixed pattern development from the first week to the third week after symptom onset. In our presentation, 21(36.2\%) patients had consolidation and of whom $5(8.6 \%)$ patients had only consolidation. Consolidation appears, which might indicate that the inflammatory exudation and atypical enlarged pneumocytes fill the intra-alveolar spaces[26]. Furthermore, a study described that there were significantly more consolidation lesions and less GGO lesions in elder patients than in younger patients[13]. However, there were no significant differences in the occurrence rate and the number of consolidation between the elder patients and younger patients in our study $(P=0.28, P=0.36$, respectively). Huang et al. also found that the intensive care unit (ICU) patients on admission had more consolidations than non-ICU patients on chest CT images[27]. In addition, some reports described that the consolidation was significantly more frequent in severe/critical patients compared with the ordinary group and considered that consolidation might be an index to reflect disease progression and consolidation increases as disease course extend $[5,13,15-17$, 23]. That may be why we found no significant difference in consolidation between the groups in our study since all the cases were ordinary type.

Interlobular septal thickening, crazy paving pattern, bronchial wall thickening, and vascular enlargement were observed in our study, but no significant difference was found between the elder group and the younger group. Xu et al[23]. considered that the thickened interlobular septa could indicate the progression of the disease. Li et al[15]. also reported that the severe/critical patients had a higher incidence of linear opacity, interlobular septal thickening, crazy-paving pattern, and bronchial wall thickening compared with the ordinary group. Pan et al[16]. suggested that the crazy-paving pattern can be as another indicator to evaluate the disease course because the lesions will absorb without crazypaving pattern on the absorption stage. Compared with the early-phase disease, the advanced-phase disease was characterized by significantly increased frequencies of reticular pattern, fibrotic streaks, subpleural line, air bronchogram, and pleural effusion[24]. In our series, some patient's first CT 
appearance already present consolidation, interlobular septal thickening, crazy-paving pattern, or bronchial wall thickening, due to they might have a long incubation period or miss the best time to the hospital. Reverse halo sign, uncommon CT imaging manifestation, was observed in 5 patients in our series, but it also can happen in pulmonary mucormycosis, invasive pulmonary aspergillosis, bacterial pneumonia, etc[28]. In our study, an uncommon halo sign was observed in 18 patients, which can be found in infectious, inflammatory, and neoplastic conditions of the lung.

In 2005, Chang et al. applied CT score to quantify the extent of the severe acute respiratory syndrome (SARS) on the basis of the lung area involved[9]. Li et al. [15]considered that the CT scores of the severe/critical patients were significantly higher than those of the ordinary patients, and CT scores were able to accurately distinguish between severe/critical patients and the ordinary patients (AUC $=0.87$ ), and at a cut-point of 7 maximizing sensitivity (80.0\%) and specificity (82.8\%). In the course of the evolution of COVID-19) pneumonia, most patients showed that the total CT score increased to approximately ten days from symptoms onset and reached its peak at 10 days, and then gradually decreased[16]. Our study used a similar approach to assess the extent of lung disease. Our finding demonstrated that the total CT score of both lungs, right lung and left lung of the elder patients were significantly higher than those of the younger patients. In addition, compared with the younger group, the older group had a higher CT score in each lobe, except for the right middle lobe. Those findings may indicate that the elder population with ordinary type may be readily to progress. The lower lobes were more inclined to be involved with higher CT score and the total CT score in the progressive stage was greater than that of the early stage in the study by Pan et al[16]. In our series, the largest lesion was usually located in the right lower lobe $(27 / 58$, $46.6 \%)$ and left lower lobe $(15 / 58,25.9 \%)$. Those findings may suggest that the lower lobes appear to have a higher CT score than other lobes due to the lower lobes are easy to be invaded by the virus and the largest lesion was often located in the lower lobe. Old age might be risk factors for poor prognosis for COVID-19 pneumonia[15, 19, 29, 30]. Combined with our findings that the elder patients had a higher CT score and more lung lobes involved than younger patients, we support the older age of patients might be a risk factor of disease severity.

There were several limitations to this study. First, we lacked follow-up CT data, due to our institution was not a designated hospital for the treatment of COVID-19 pneumonia. We will collect more follow-up CT data to describe the progress and prognosis of the disease in the future. Secondly, the analysis was limited to patients with ordinary 2019-nCoV pneumonia, the sample size is relatively small. Thirdly, the CT scanning parameters of different institutions were not consistent, some uncommon imaging findings such as tiny pulmonary nodules might be underestimated on CT images with $5 \mathrm{~mm}$ slice thickness or more.

In summary, ordinary COVID-19 pneumonia on chest CT generally manifested as multiple GGO and consolidation in the bilateral lung, with peripheral or mixed distribution, and lower lobe predilection. There were more lung lobes involved in the elder group (age $\geq 50 y e a r s)$ than the younger group (age $<50$ years). The CT score of each lobe in elder patients was higher than younger patients, except for the middle lobe of the right lung, the lesions with a maximum diameter larger than $5 \mathrm{~cm}$ were more likely to occur in the 
elder group than the younger group. The older age may be an indicative factor for disease progression in ordinary type, clinicians must pay close attention to this population.

\section{Declarations}

\section{Acknowledgements}

Not applicable

\section{Ethical standards}

All procedures performed in studies involving human participants were in accordance with the ethical standards of the institutional and/or national research committee and with the 1964 Declaration of Helsinki and its later amendments or comparable ethical standards. This article does not contain any studies with animals performed by any of the authors. Informed consent was waived from all individual participants included in the study.

\section{Conflict of interest}

The authors declare that they have no conflict of interest.

\section{References}

[1] Zhu N, Zhang D, Wang W, Li X, Yang B, Song J, et al (2020) A novel coronavirus from patients with pneumonia in China, 2019. N Engl J Med. 382:727-33. https:// doi: 10.1056/NEJMoa2001017

[2] Gorbalenya AE, Baker SC, Baric RS, et al (2020) Severe acute respiratory syndrome-related coronavirus: The species and its viruses - a statement of the Coronavirus Study Group. bioRxiv. https://doi.org/10.1101/2020.02.07.937862

[3] World Health Organization. WHO Director-General's remarks at the media briefing on 2019-nCoV on 11 February 2020. https://www.who.int/dg/speeches/detail/who-director-general-s-remarks-at-the-mediabriefing-on-2019-ncov-on-11-february-2020

[4] Xie X, Zhong Z, Zhao W, Zheng C, Wang F, Liu J (2020) Chest CT for Typical 2019-nCoV Pneumonia: Relationship to Negative RT-PCRTesting. Radiology. 200343. https://doi:10.1148/radiol.2020200343

[5] Zu ZY, Jiang MD, Xu PP, Chen W, Ni QQ, Lu GM, et al (2020) Coronavirus Disease 2019 (COVID-19): A Perspective from China. Radiology. 200490. https:// doi:10.1148/radiol.2020200490

[6] Fang Y, Zhang H, Xie J, Lin M, Ying L, Pang P, et al (2020) Sensitivity of Chest CT for COVID-19 Comparison to RT-PCR. Radiology. 200432. https:// doi: 10.1148/radiol.2020200432 
[7] Ai T, Yang Z, Hou H, Zhan C, Chen C, Lv W, et al (2020) Correlation of Chest CT and RT-PCR Testing in Coronavirus Disease 2019 (COVID-19) in China: A Report of 1014 Cases. Radiology. 200642. https:// doi: $10.1148 /$ radiol.2020200642

[8] General Office of National Health Committee. Office of State Administration of Traditional. Notice on the issuance of a program for the diagnosis and treatment of novel coronavirus (2019-nCoV) infected pneumonia(trial seventh edition)[EB/OL].

http://www.nhc.gov.cn/yzygj/s7652m/202003/a31191442e29474b98bfed5579d5af95. shtml. accessed 4 March 2020

[9] Chang YC, Yu CJ, Chang SC, Galvin JR, Liu HM, Hsiao CH, et al (2005) Pulmonary sequelae in convalescent patients after severe acute respiratory syndrome: evaluation with thin-section CT. Radiology. 236 (3):1067-75. https:// doi:10.1148/radiol.2363040958

[10] Wang D, Hu B, Hu C, Zhu F, Liu X, Zhang J, et al (2020) Clinical Characteristics of 138 Hospitalized Patients With 2019 Novel Coronavirus-Infected Pneumonia in Wuhan, China. JAMA. https:// doi: $10.1001 /$ jama.2020.1585

[11] Sun P, Qie S, Liu Z, Ren J, Li K, Xi J (2020) Clinical characteristics of 50466 hospitalized patients with 2019-nCoV infection. J Med Virol. https:// doi: 10.1002/jmv.25735

[12] Yang W, Cao Q, Qin L, Wang X, Cheng Z, Pan A, et al (2020) Clinical characteristics and imaging manifestations of the 2019 novel coronavirus disease (COVID-19): A multi-center study in Wenzhou city. J Infect. https:// doi: 10.1016/j.jinf.2020.02.016

[13] Song F, Shi N, Shan F, Zhang Z, Shen J, Lu H, et al (2020) Emerging Coronavirus 2019-nCoV Pneumonia. Radiology. 295(1): 210-217. https:// doi: 10.1148/radiol.2020200274

[14] Chung M, Bernheim A, Mei X, Zhang N, Huang M, Zeng X, et al (2020) CT Imaging Features of 2019 Novel Coronavirus (2019-nCoV). Radiology. 295(1):202-207. https:// doi: 10.1148/radiol.2020200230

[15] Li K, Wu J, Wu F, Guo D, Chen L, Fang Z, et al (2020) The Clinical and Chest CT Features Associated with Severe and Critical COVID-19 Pneumonia. https:// doi: 10.1097/RLI.0000000000000672

[16] Pan F, Ye T, Sun P, Gui S, Liang B, Li L, et al (2020) Time Course of Lung Changes On Chest CT During Recovery From 2019 Novel Coronavirus (COVID-19) Pneumonia. Radiology. 200370. https://doi: $10.1148 /$ radiol.2020200370

[17] Pan Y, Guan H. Imaging changes in patients with 2019-nCov (2020) Eur Radiol. https:// doi: 10.1007/s00330-020-06713-z

[18] Pan Y, Guan H, Zhou S, Wang Y, Li Q, Zhu T, et al (2020) Initial CT findings and temporal changes in patients with the novel coronavirus pneumonia (2019-nCoV): a study of 63 patients in Wuhan, China. Eur Radiol.https:// doi: 10.1007/s00330-020-06731-x 
[19] Shi H, Han X, Jiang N, Cao Y, Alwalid O, Gu J, et al (2020) Radiological findings from 81 patients with COVID-19 pneumonia in Wuhan, China: a descriptive study. Lancet Infect Dis. https://doi:

10.1016/S1473-3099(20)30086-4

[20] Shi H, Han X, Zheng C (2020) Evolution of CT Manifestations in a Patient Recovered from 2019 Novel Coronavirus (2019-nCoV) Pneumonia in Wuhan, China. Radiology. 295(1): 20. https://doi:

$10.1148 /$ radiol.2020200269

[21] Wu J, Wu X, Zeng W, Guo D, Fang Z, Chen L, et al (2020) Chest CT Findings in Patients with Corona Virus Disease 2019 and its Relationship with Clinical Features. Invest Radiol. https://doi:

10.1097/RLI.0000000000000670

[22] Xu X, Yu C, Qu J, Zhang L, Jiang S, Huang D, et al (2020) Imaging and clinical features of patients with 2019 novel coronavirus SARS-CoV-2. Eur J Nucl Med Mol Imaging. https:// doi: 10.1007/s00259020-04735-9

[23] Xu YH, Dong JH, An WM, Lv XY, Yin XP, Zhang JZ, et al (2020) Clinical and computed tomographic imaging features of novel coronavirus pneumonia caused by SARS-CoV-2. J Infect. https:// doi: 10.1016/j.jinf.2020.02.017

[24] Zhou S, Wang Y, Zhu T, Xia L (2020) CT Features of Coronavirus Disease 2019 (COVID-19) Pneumonia in 62 Patients in Wuhan, China. AJR Am J Roentgenol. 1-8. https:// doi:

10.2214/AJR.20.22975

[25] Bernheim A, Mei X, Huang M, Yang Y, Fayad ZA, Zhang N, et al (2020) Chest CT Findings in Coronavirus Disease-19 (COVID-19) Relationship to Duration of Infection. Radiology. 200463. https:// doi: 10.1148/radiol.2020200463

[26] Xu Z, Shi L, Wang Y, Zhang J, Huang L, Zhang C, et al (2020) Pathological findings of COVID-19 associated with acute respiratory distress syndrome. Lancet Respir Med. https://doi:10.1016/S22132600(20)30076-X

[27] Huang C, Wang Y, Li X, Ren L, Zhao J, Hu Y, et al (2020) Clinical features of patients infected with 2019 novel coronavirus in Wuhan, China.Lancet.395(10223):497-506.https://doi:10.1016/S01406736(20)30183-5

[28] Thomas R, Madan R, Gooptu M, Hatabu H, Hammer MM (2019) Significance of the Reverse Halo Sign in Immunocompromised Patients. AJR Am J Roentgenol.213(3):549-54.https://doi:

10.2214/AJR.19.21273

[29] Liu Y, Yang Y, Zhang C, Huang F, Wang F, Yuan J, et al (2020) Clinical and biochemical indexes from 2019-nCoV infected patients linked to viral loads and lung injury. Sci China Life Sci. 63(3):364-74. https:// doi: 10.1007/s11427-020-1643-8 
[30] Zhao W, Zhong Z, Xie X, Yu Q, Liu J (2020) Relation Between Chest CT Findings and Clinical Conditions of Coronavirus Disease (COVID-19) Pneumonia: A Multicenter Study.AJR Am J Roentgenol.16. https:// doi: 10.2214/AJR.20.22976

\section{Tables}

Table 1. Clinical characteristics and laboratory findings in 58 patients with ordinary COVID-19 pneumonia

\begin{tabular}{|c|c|}
\hline Clinical data & $\begin{array}{l}\text { Number }(\%) \\
n=58\end{array}$ \\
\hline Age (y) & $44.7 \pm 13.8$ \\
\hline Onset of illness to hospital (d) & $3(1.0-5.3)$ \\
\hline \multicolumn{2}{|l|}{ Sex } \\
\hline Male & $28 / 58(48.3 \%)$ \\
\hline Female & $30 / 58(51.7 \%)$ \\
\hline \multicolumn{2}{|l|}{ Symptoms and Signs } \\
\hline \multicolumn{2}{|l|}{ Temperature } \\
\hline Normal & $15 / 58(25.9 \%)$ \\
\hline Fever & $43 / 58(74.1 \%)$ \\
\hline 37.3-38.0 C & $27 / 58(46.6 \%)$ \\
\hline $38.1-39.0 \mathrm{C}$ & $15 / 58(25.9 \%)$ \\
\hline$>39.0 \mathrm{C}$ & $1 / 58(1.7 \%)$ \\
\hline Cough & $41 / 58(70.7 \%)$ \\
\hline Expectoration & $5 / 58(8.6 \%)$ \\
\hline Headache & $4 / 58(6.9 \%)$ \\
\hline Diarrhea & $2 / 58(3.4 \%)$ \\
\hline Generalised weakness & $5 / 58(8.6 \%)$ \\
\hline Dizziness & $4 / 58(6.9 \%)$ \\
\hline Pharyngalgia & $2 / 58(3.4 \%)$ \\
\hline Decreased oxygen saturation & $2 / 58(3.4 \%)$ \\
\hline \multicolumn{2}{|l|}{ Laboratory findings } \\
\hline Leucopenia & $5(8.6 \%)$ \\
\hline Lymphopenia & $35(60.3 \%)$ \\
\hline Lymphocyte ratio decreased & $22(37.8 \%)$ \\
\hline Neutrophil ratio increased & $12(20.7 \%)$ \\
\hline C-reactive protein increased & $35(60.3 \%)$ \\
\hline
\end{tabular}

Data are n (\%), mean (SD), median (IQR) 
Table 2. CT imaging findings in 58 patients with ordinary COVID-19 pneumonia

Page 13/18 


\begin{tabular}{|c|c|c|}
\hline Imaging findings & $\begin{array}{c}\text { No. of patients }(\%) \\
(\mathrm{n}=58)\end{array}$ & No. of lesions $(\%)(n=634)$ \\
\hline \multicolumn{3}{|l|}{ Distribution } \\
\hline Peripheral & $26(44.8 \%)$ & $97(15.3 \%)$ \\
\hline Central & $2(3.5 \%)$ & $2(0.3 \%)$ \\
\hline Mixed & $30(51.7 \%)$ & $535(84.4 \%)$ \\
\hline Single & $10(17.2 \%)$ & $10(1.6 \%)$ \\
\hline Multiple & $48(82.8 \%)$ & $624(98.4 \%)$ \\
\hline \multicolumn{3}{|l|}{ Involved lung lobes and segments } \\
\hline Right upper lobe & $39(67.2 \%)$ & $141(22.2 \%)$ \\
\hline Apical segment & $8(13.8 \%)$ & $26(4.1 \%)$ \\
\hline Posterior segment & $31(53.4 \%)$ & $71(11.2 \%)$ \\
\hline Anterior segment & $23(39.7 \%)$ & $44(6.9 \%)$ \\
\hline Right middle lobe & $26(44.8 \%)$ & $46(7.3 \%)$ \\
\hline Lateral segment & $19(32.8 \%)$ & $27(4.3 \%)$ \\
\hline Medial segment & $14(24.1 \%)$ & $19(3.0 \%)$ \\
\hline Right lower lobe & $48(82.8 \%)$ & $187(29.5 \%)$ \\
\hline Dorsal segment & $30(51.2 \%)$ & $50(7.9 \%)$ \\
\hline Medial basal segment & $17(29.3 \%)$ & $19(3.0 \%)$ \\
\hline Anterior basal segment & $14(24.1 \%)$ & $15(2.4 \%)$ \\
\hline Lateral basal segment & $20(34.5 \%)$ & $29(4.6 \%)$ \\
\hline Posterior basal segment & $32(55.2 \%)$ & $74(11.7 \%)$ \\
\hline Left upper lobe & $35(60.3 \%)$ & $108(17.0 \%)$ \\
\hline Apical-posterior segment & $27(46.6 \%)$ & $58(9.1 \%)$ \\
\hline Anterior segment & $7(12.1 \%)$ & $11(1.7 \%)$ \\
\hline Superior lingual segment & $13(22.4 \%)$ & $15(2.4 \%)$ \\
\hline Inferior lingual segment & $17(29.3 \%)$ & $24(3.8 \%)$ \\
\hline Left lower lobe & $36(62.1 \%)$ & $152(24.0 \%)$ \\
\hline Dorsal segment & $26(44.8 \%)$ & $40(6.3 \%)$ \\
\hline Anterior-medial basal segment & $20(34.5 \%)$ & $29(4.6 \%)$ \\
\hline Lateral basal segment & $22(37.9 \%)$ & $27(4.3 \%)$ \\
\hline Posterior basal segment & $29(50.0 \%)$ & $56(8.8 \%)$ \\
\hline \multicolumn{3}{|l|}{ Pattern of lesion } \\
\hline GGO & $53(91.4 \%)$ & $451(71.1 \%)$ \\
\hline Consolidation & $21(36.2 \%)$ & $157(24.8 \%)$ \\
\hline Pleural thickening & $5(8.6 \%)$ & $7(1.1 \%)$ \\
\hline Subpleural line & $6(19.0 \%)$ & $9(1.4 \%)$ \\
\hline Fibrous stripes & $5(8.6 \%)$ & $10(1.6 \%)$ \\
\hline Interlobular septal thickening & $12(20.7 \%)$ & $37(5.8 \%)$ \\
\hline Crazy-paving pattern & $9(15.5 \%)$ & $28(4.4 \%)$ \\
\hline Bronchial wall thickening & $7(12.1 \%)$ & $15(2.4 \%)$ \\
\hline Vascular enlargement & $8(13.8 \%)$ & $12(1.9 \%)$ \\
\hline
\end{tabular}




$\begin{array}{lcl}\text { Halo sign } & 18(31.0 \%) & 44(6.9 \%) \\ \text { Reverse halo sign } & 5(8.6 \%) & 7(1.1 \%) \\ \text { Air bronchogram sign } & 10(17.2 \%) & 15(2.4 \%)\end{array}$

Data are $\mathrm{n}(\%) . \mathrm{GGO}=$ ground glass opacity

Table 3. The difference of CT features between group 1 ( $<50$ years) and group 2 (50 $\geq$ years)

\begin{tabular}{|c|c|c|c|c|}
\hline Parameter & Total $(n=58)$ & Group $1(\mathrm{n}=33)$ & Group $2(n=25)$ & $P$ Value \\
\hline \multicolumn{5}{|l|}{ Radiological score } \\
\hline Bilateral lungs & $5(2-7.25)$ & $3(2-6)$ & $6(4.5-9.5)$ & 0.003 \\
\hline Right lung & $3(1.75-4.25)$ & $2(1-3.5)$ & $3(2.5-5.5)$ & 0.012 \\
\hline Left lung & $2(1-3)$ & $1(0-2)$ & $3(2-4.5)$ & 0.002 \\
\hline Right upper lobe & $1(0-1.25)$ & $1(0-1)$ & $1(1-2)$ & 0.004 \\
\hline Right middle lobe & $0(0-1)$ & $0(0-1)$ & $0(0-1)$ & 0.979 \\
\hline Right lower lobe & $1(1-2)$ & $1(1-2)$ & $2(1-3)$ & 0.043 \\
\hline Left upper lobe & $1(0-1)$ & $0(0-1)$ & $1(0.5-2)$ & 0.001 \\
\hline Left lower lobe & $1(0-2)$ & $1(0-2)$ & $2(1-3)$ & 0.031 \\
\hline \multicolumn{5}{|l|}{ CT pattern } \\
\hline (No. of patients [\%] ) & $21(36.2 \%)$ & $10(30.3 \%)$ & $11(44.0 \%)$ & 0.282 \\
\hline $\begin{array}{l}\text { Consolidation } \\
\text { (No. of lesions) }\end{array}$ & $0(0-1)$ & $0(0-1)$ & $0(0-1.5)$ & 0.36 \\
\hline \multicolumn{5}{|l|}{$\begin{array}{l}\text { Lesion distribution } \\
\text { (No. of lesions) }\end{array}$} \\
\hline Central & $1(0-3.25)$ & $1(0-3.5)$ & $1(0-3)$ & 0.62 \\
\hline Peripheral & $4(2-13)$ & $3(1-7)$ & $7(3-17.5)$ & 0.022 \\
\hline Number of involved lobes & $4(2.0-4.25)$ & $3(1.5-4)$ & $4(3-5)$ & 0.024 \\
\hline Number of involved segments & $5(2.0-10.0)$ & $4(1.5-9.5)$ & $7(3.5-11)$ & 0.120 \\
\hline $\begin{array}{l}\text { Maximum diameter } \\
\text { (No. of patients [\%]) }\end{array}$ & $31(53.4 \%)$ & $12(36.4 \%)$ & $19(76 \%)$ & 0.003 \\
\hline
\end{tabular}

Data are n (\%), median (IQR)

Figures 

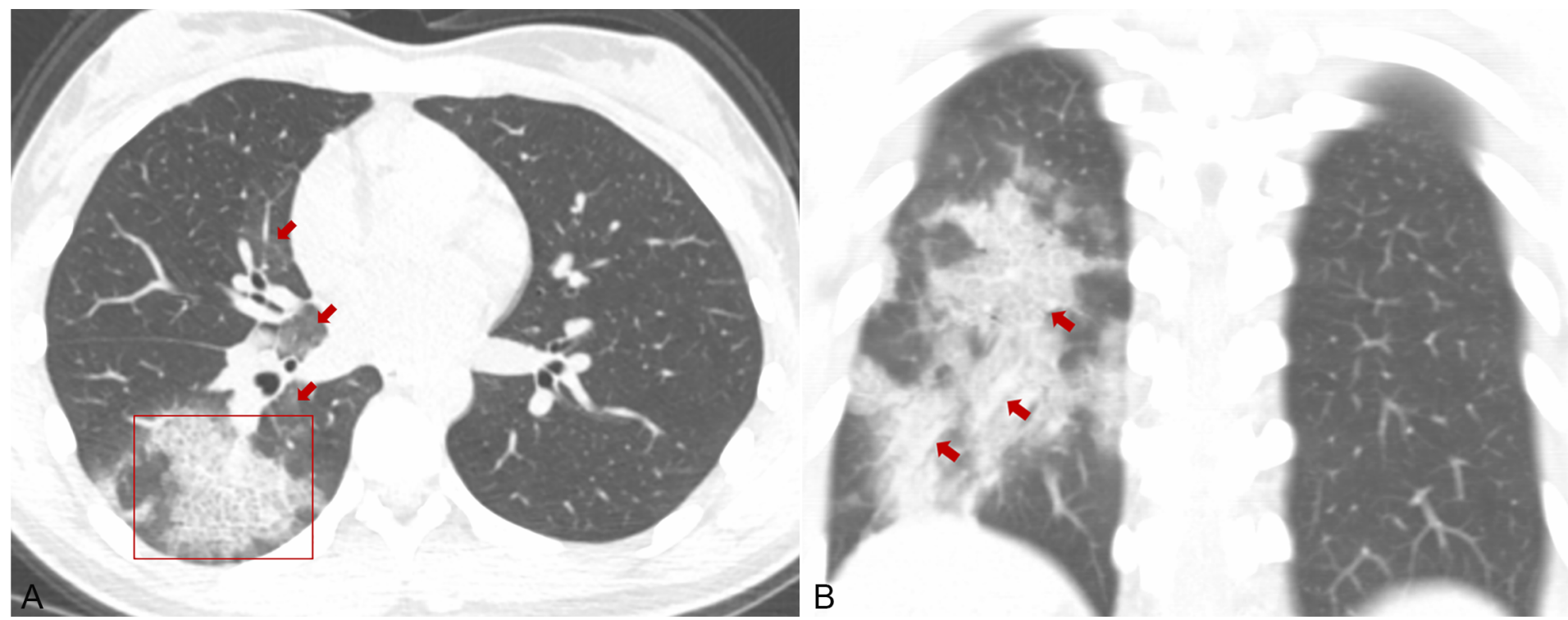

\section{Figure 1}

CT features of ordinary COVID-19 pneumonia. A. An axial CT image without contrast demonstrates crazypaving pattern (GGO with intralobular septal thickening, red box), GGO (red arrow). B. A coronal CT image shows vascular enlargement (red arrow). COVID-19, corona virus disease 2019; GGO, ground-glass opacity.

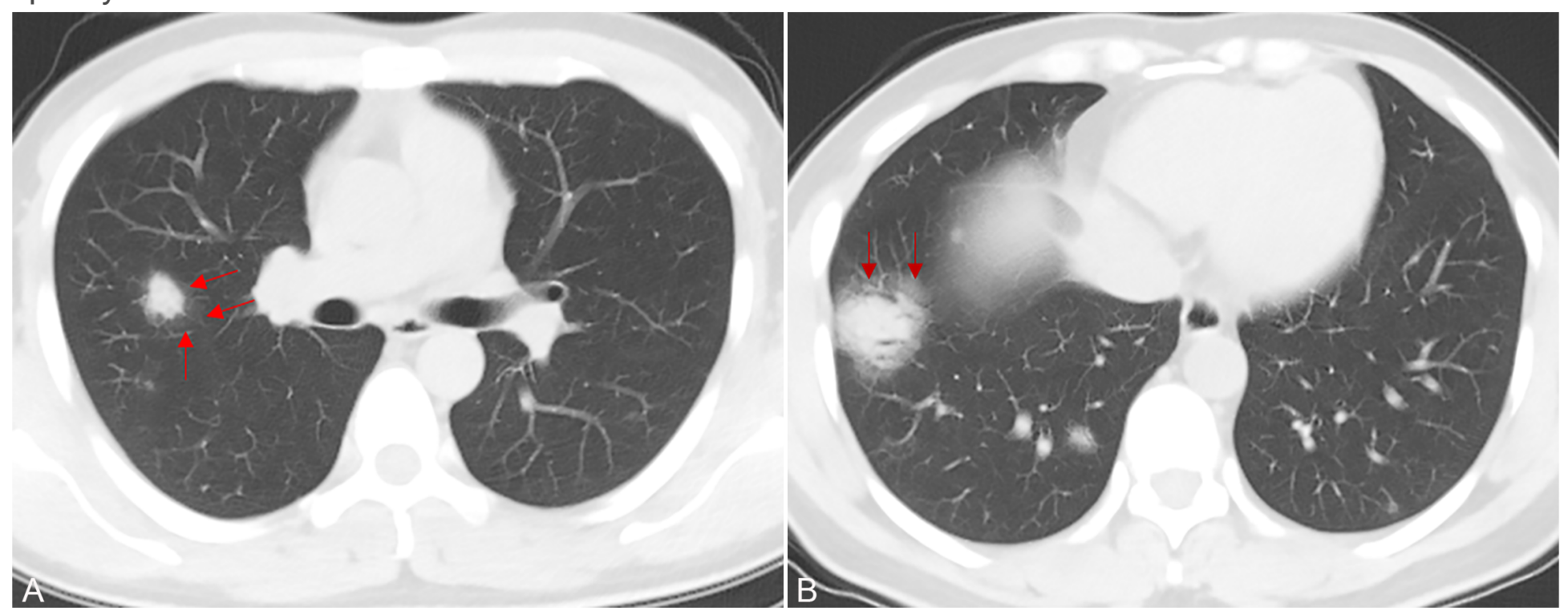

Figure 2

Various lesions of an included case. Axial CT images show multiple consolidations in bilateral lungs, consolidation with "halo sign" (red arrow) can be observed in right lung. 


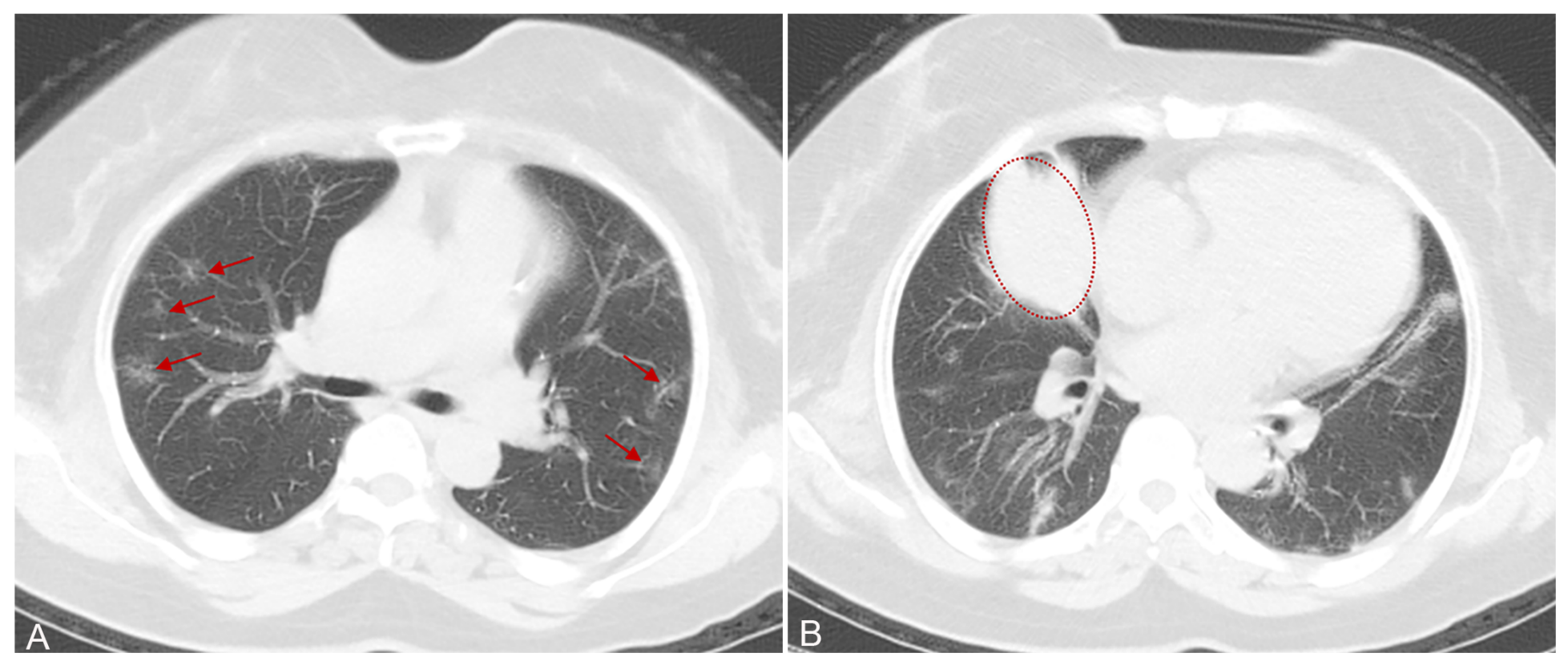

Figure 3

Axial CT images demonstrate multiple lesions of subpleural GGO (red arrow) with consolidation (red circle). GGO, ground-glass opacity. 


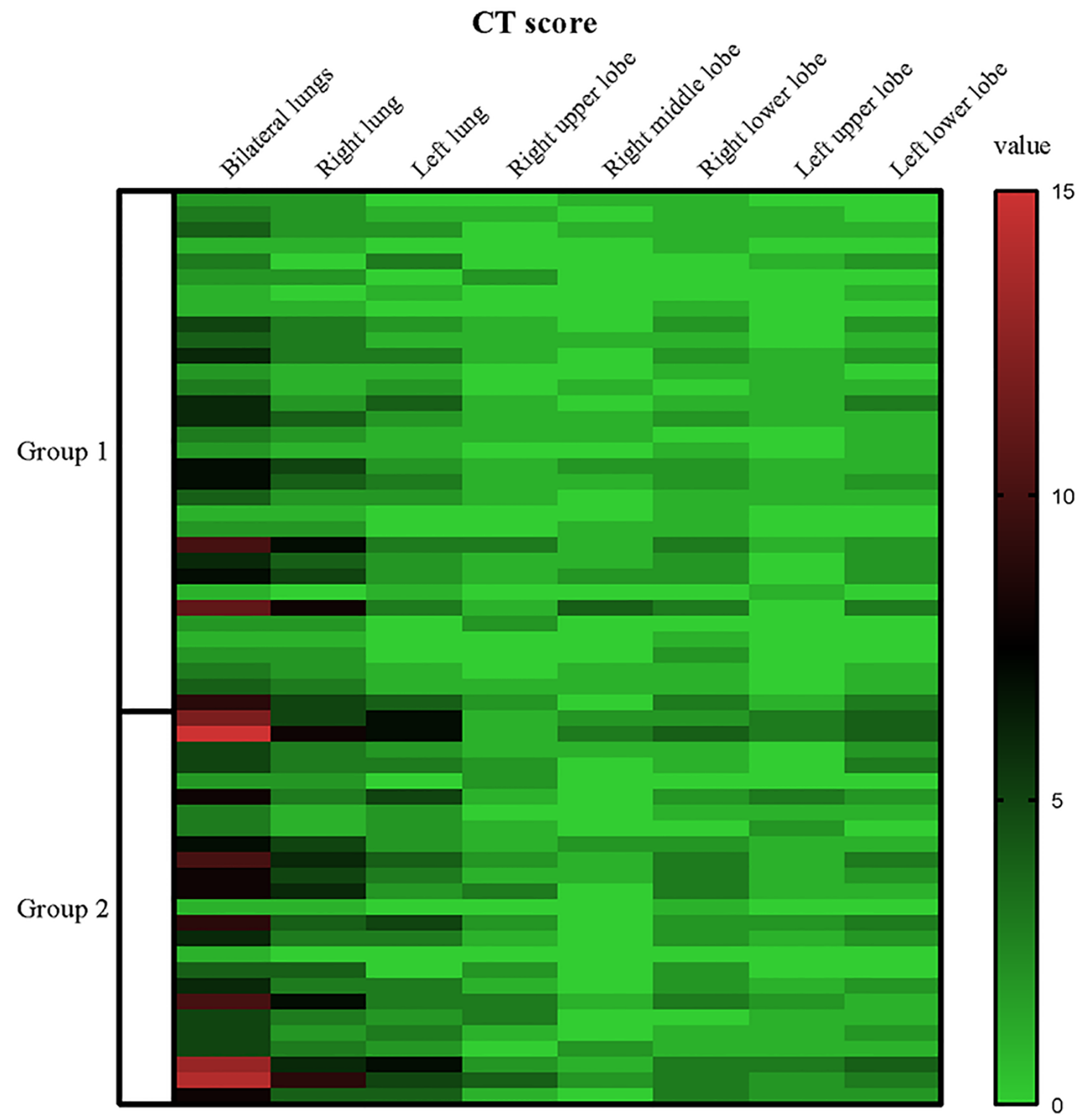

Figure 4

58 patients with ordinary COVID-19 pneumonia are grouped by age of the patient: group 1 ( $<50$ years, $n=33)$, group 2 ( $50 \geq$ years, $n=25$ ). The CT score of the bilateral lungs, right lung, left lung and all lobes in every patient is shown by heatmap, and the colors in the heatmap indicate the value of the CT score. 